\title{
ONGOING COVID-19 SYNDROME AND POST-COVID-19 SYNDROME: LONG-TERM SYMPTOMS AND RESIDUAL CHANGES AFTER SARS-COV-2 INFECTION
}

\author{
Kosta V. Kostov* \\ INSPIRO Medical Center and INSPIRO Respiratory Medicine Foundation, Sofia, Bulgaria
}

Recently, in the time SARS-CoV-2 pandemic, there is increasing interest among the medical community in post-COVID-19 residual symptoms including structural and functional changes in different organs and systems. The symptom characteristic and parameters of these changes in the period after illness are in the process of registration and complete clarification, and the first results were published. It becomes clear that symptoms of COVID-19 (over 30 in number) even among those with a "mild form" of the disease remain for a long time after the acute phase in a large number of patients. More data are needed on the somatic, mental and emotional characteristics of patients who have undergone COVID-19, the so-called ,prolonged COVID-19 syndrome“, despite the lack of a generally accepted definition.. Knowledge of its clinical characteristic will help physicians to recognize its manifestations, and the healthcare system to prepare for its prevention and treatment. Biomed Rev 2020; 31: 117-123

Keywords: CoV-2, ongoing COVID-19 syndrome, post-COVID-19 syndrome

\section{INTRODUCTION}

Clarifying the characteristics of prolonged COVID-19 syndrome becomes even more necessary given the assumptions that those who have undergone SARS-CoV-2 (hereafter, CoV2) infection are at least 10 times more than those diagnosed and known to the medical community. The majority of the infected have gone through a mild form and stayed at home, but all, regardless of the severity of disease, may have symptoms that persist for weeks and months after and require medical care.

\section{ACUTE INFECTION WITH COV-2}

Symptoms during the active course of COVID-19

Listed in a group without claims of absolute completeness, symptoms that were recorded during COVID-19 and reported in the scientific literature are: shortness of breath, fatigue, increased body temperature (up to $37.9^{\circ} \mathrm{C}$ ), chills $\left(\geq 38^{\circ} \mathrm{C}\right.$ ),

\footnotetext{
*Correspondence to: Dr Kosta V. Kostov, INSPIRO Medical Center, 4 Petar Protich Street, BG-1000 Sofia, Bulgaria
} E-mail: korokostov@gmail.com 
cough, cold nose, sneezing, sore throat, muscle aches, joint pains, loss of sense of smell, loss of taste, headache, dizziness, diarrhea, nausea, vomiting, red spots on the toes or other anatomical areas of the legs, feeling of pain or heat in the lung, ear pain, tightness of the chest, back pain between the blades, palpitations, rapid cardiac activity at rest, eye problem, rapid weight loss, sensation of burning in the trachea, tides of heat on the body, rashes, chills, loss of concentration, cognitive problems (memory, information processing, processes of cognition, decision-making, language capabilities, etc), sleep disorders (1-5).

One of the essential characteristics of symptoms in the acute phase is the wavy course in some of the sick, at tides, alternating some symptoms with other symptoms, through phases of slight improvement and subsequent deterioration. Not all patients register classic starter symptoms such as fever, shortness of breath and cough. In the majority of patients, cough comes a little later, in others there is no fever, and physical weakness more often precedes and is more palpable than the breath, which also comes later. In one part of the patients at first there are atypical symptoms such as instability of blood pressure or pain in the heart area. In my decadeslong practice as a pulmonologist, I have never encountered such a disease, with such "artistic" behavior, more talented even than tuberculosis, which we call "the great actress". The good news is that most of my patients, whom I controlled in home treatment mode, recovered almost completely, with little residual damages and symptoms. This cannot be said conclusively for those patients who have had a severe illness, went through the intensive care units that will need longerterm monitoring, treatment and rehabilitation. In the process of active infection, in one of the studies, $97 \%$ of patients had more than five of the listed symptoms, with an average number of 14 symptoms (11-17), with feeling tired and shortness of blown out being the most commonly reported symptoms, resp. $94.9 \%$ (fatigue) and $89.5 \%$ (shortness of breath) (1).

\section{PROLONGED (POST-) COVID-19 SYNDROME Symptoms after acute illness of COVID-19 Late inflammatory and virological consequences}

Monitoring of symptoms from the acute period of the disease shows that they decrease significantly in number, with 2-3 months after illness still the most common residual symptoms are fatigue and shortness of breath $(1,6)$. In one study, only $0.7 \%$ of patients were completely asymptomless after illness, and there were individual patients $(2 \%)$ who reported more symptoms from the period of illness (1). The summarized published data from patient follow-up show that two months after hospital treatment, no more than $13 \%$ of discharges are completely asymptom, about $30 \%$ of patients have 1 or 2 residual symptoms, over $50 \%$ have 3 or more symptoms, in total about $2 / 3$ of patients have residual symptoms after the symptom period. The most commonly recorded symptoms during and after COVID-19 were shortness of breath and fatigue $(1,7,8)$. Commonly reported symptoms are also muscle and joint pain, loss of taste and sense of smell $(6,8)$. This shows that in the period after illness there is only a partial recovery of symptoms and the health status of the sick, and very few patients have a full recovery even months after the acute phase of the disease. Residual symptomatology has a significant dependence on age, hospital stay, severe course and presence of shortness of breath at the beginning of the disease (8).

So far, there is no well-established and generally accepted definition, as well as biomarkers to prove prolonged COVID-19 syndrome, called by some colleagues „Long Covid“; so it is determined by the symptoms and the time frame. There are reasons to define three concepts, which are stages in the course of COVID-19:

(i) acute COVID-19: duration of symptoms up to 4 weeks

(ii) post-acute (ongoing) symptomatic COVID-19: duration of symptoms from 4 to 12 weeks after the onset of the disease, and

(iii) prolonged (post-) COVID-19: duration of symptoms $>12$ weeks after the onset of the disease, which cannot be explained by another disease and the duration of which is not yet definitively known (9).

It should be noted that the development of symptoms within the post-acute and prolonged Covid-19 is not directly dependent on the severity of the course of acute Covid-19 or the place of treatment - in or out of hospital. Symptoms can vary over time, disappear and reappear, as well as change in intensity.

In telephone follow-up of patients with outpatiently proven CoV-2 infection, $35 \%$ of those who had symptoms during infection still had such two weeks or more after the positive test, with symptoms showing a proportional relationship with the age of patients and the most pronounced were in those $>50$ years and less prominent in those $<35$ years (10). Similarly, data from British authors, who tracked 100 hospitalized patients who went through a general and intensive care unit and found that 4-8 weeks after discharge from the hospital, 
$72 \%$ of patients who underwent intensive care units and $60 \%$ went through general wards had increasingly complained of shortness of breath and fatigue (11).

Organ symptomatology after COVID-19 is primarily controlled by the respiratory system, heart and nervous system, but although less often the kidneys, liver, pancreas, spleen, even skin and mucous membranes are also affected, possibly a consequence of the direct invasion of the virus into tissues, pronounced inflammation, immunological imbalance, cytokine storm and blood clotting disorders with subsequent thromboembolisms in the most severe cases of the disease. Despite the small number of patients with residual health problems against the background of all infected and tenfold more undetected due to asymptomatic flow, health systems around the world will face the challenge of dealing with this problem in hundreds of thousands of patients worldwide. In Bulgaria, this share for now is several hundred, but with a longer and more aggressive course of the epidemic, it is possible to become several thousand. It should be considered that residual somatic and functional problems are also detected in mild forms of COVID-19 and can last for an extended period of time, disrupting the quality of life of the sick. Although the lung is first affected by the disease, in many patients it is not the most affected organ, and this gives us the right to define the disease not just as a respiratory disease, but as a systemic disease. Let's not forget also that patients with a more severe course will remain for a different period of time with a different pronounced imbalance of the immune system and an increased risk of infection with other pathogens.

\section{Cardiovascular consequences}

Myocardial impairment (cardiomyopathy) is clinically objectified with an increased level of troponin in serum in more severe cases of COVID-19. In infected with CoV-2, inflammatory changes in the myocardium (myocarditis) often occur with arrhythmia. Data from magnetic resonance imaging of the heart in 100 patients, carried out on the 71 day after disease from COVID-19 show that in $78 \%$ there is a heart effect, and in $60 \%$ the process of myocardial involvement is still active. Myocarditis and myocardial inflammatory changes were recorded by magnetic resonance imaging and in $12(46 \%)$ of 26 elite American athletes, within 12-53 days after infection with $\mathrm{CoV}-2$, but went through the infection without symptoms (12). These data raise concerns about a future increase in heart failure cases, especially in older and patients with accompanying diseases.

\section{Neurological consequences}

Damage to the nervous system occurs after viral pennetration in the nervous tissue, as well as from the direct invasion by the virus of neurons. The most common neurological residual changes that are recorded in patients with COVID-19 are headache, dizziness, hemosensory dysfunction (anosmia and agenesis), peripheral neuropathies, sleep disorders, deliriums (in older patients). Stroke is a rare manifestation in the acute phase of infection, but in the post-acute period, up to 2-3 months after illness, cases of encephalitis, seizures, significant mood swings, confusion, memory and cognitive disorders, physical discomfort, also observed in previous coronavirus pandemics (13).

In a study of British colleagues who used a cognitive questionnaire in about 84,000 participants, they found a significant volume of cognitive impairment even in patients who went through the infection slightly, without shortness of breath and without any other residual symptomatology. This builds the hypothesis that COVID-19 has a systemic effect on cognitive functions (14). There are concerns that COVID-19 increases the risk of developing Parkinson's disease and Alzheimer's disease.

\section{Emotional consequences}

Months of isolation led to lasting changes in the emotional state of patients. Cases of feelings of loneliness and isolation, hopelessness, physical discomfort, anxiety, exhaustion, physical exhaustion, which can be summarized by the term "chronic fatigue syndrome" which proceeds with chronic emotional instability and physical deficiency, and does not allow for full-fledged work, have increased. Those suffering from COVID-19 have an increased risk of developing residual depression, fearful experiences, insecurity, post-traumatic stress and misuse of toxic substances (15). Young patients are more prone to emotional consequences than patients $>60$ years $(11,16)$.

\section{Pulmonary consequences}

The lung is the closest target organ in the path of the virus, and all pathogenic processes induced by $\mathrm{CoV}-2$ are played out most severely in the pulmonary parenchyma, including alveolitis, caused by immunological imbalance and cytokine storm, blood vessel endothelial damage, thrombosis and thromboembolism, destruction of alveolo-capillary membrane, secondary bacterial infections, etc.

In a study, tracking 55 patients with COVID-19 at the 
third month after discharge from hospital, 64\% (35) still have symptoms, and $71 \%$ (39) still have radiological changes with a characteristic of outbreak fibrosis and interstitial seals that can be associated with persistent symptomatology and functional changes persisting in $25 \%$ of patients with reduced diffusion capacity (17). In another study with 57 patients, 30 (53\%) diffusion capacity changes are recorded 30 days after discharge from hospital. Functional changes in the lung go to a proportion of patients with cardiac residual changes, and this further complicates their functional status (18). Of course, the data show that over time, some of the residual changes are absorbed and the percentage of patients affected declines (Fig. 1). Some of the residual damages are due to therapeutic interventions, e.g. intubation, but the majority are a direct consequence of viral aggression.

In a large prospective cohort study of 101 patients after hospitalization for COVID-19, $76 \%$ of them reported residual complaints and impaired quality of life 3 months after the onset of symptoms. The most common residual changes are in quality of life, the presence of dyspnea and cough, and these changes are not affected by the presence or absence of accompanying diseases, which speaks of a direct pathogenic effect of the virus and the damages that occurred (19).

\section{Symptoms after suffering from a critical form of COVID-19 pneumonia \\ COVID-19 syndrome after treatment in an intensive care unit (ICU)}

A particular form of the syndrome after getting sick from
COVID-19 is the symptom complex, which develops after a critical form of illness and carried out treatment in an ICU and can lead to a significant violation of the quality of life of patients after the acute phase of the disease. In the scientific literature it is defined by the term "Post Intensive Care Syndrome", which we can call "COVID-19 syndrome after treatment in an intensive care unit" - a spectrum of somatic (physical deficiency), emotional and mental residual changes that characterize the condition of patients who survived the disease after treatment in an ICU (20). Among the residual complaints are muscle weakness, problems in cognitive functions (memory, learning, reasoning alike), disturbed sleep, depression, fear, and post-traumatic stress. All these residual changes affect not less than $30 \%-50 \%$ of patients who have gone through intensive care units, and cognitive disorders even up to $80 \%$ of this group of patients. Paralytic manifestations may also be registered. All described disorders of "COVID-19 syndrome after treatment in an intensive dacre unit" can proceed individually or in different combinations. Peripheral paralysis within this syndrome most often occurs as a result of mixed motor and axonal polyneuropathy (critical illness polyneuropathy) and myopathy (critical illness myopathy), conditioned by a critical course of the disease. Guillain-Barre syndrome is a rare complication of COVID-19, and may occur a few days after the first respiratory complaints (21). Often neurological manifestations can go unrecognized within severe respiratory insufficiency.

Diagnosis of residual structural and functional disorders of the respiratory system after COVID-19 is carried out

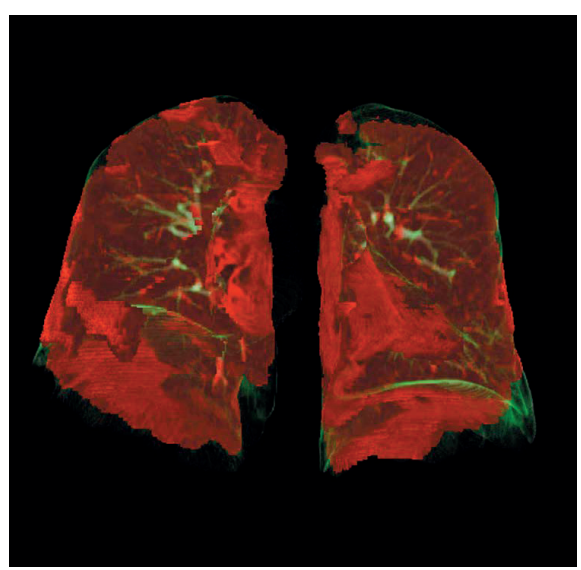

Start of COVID-19

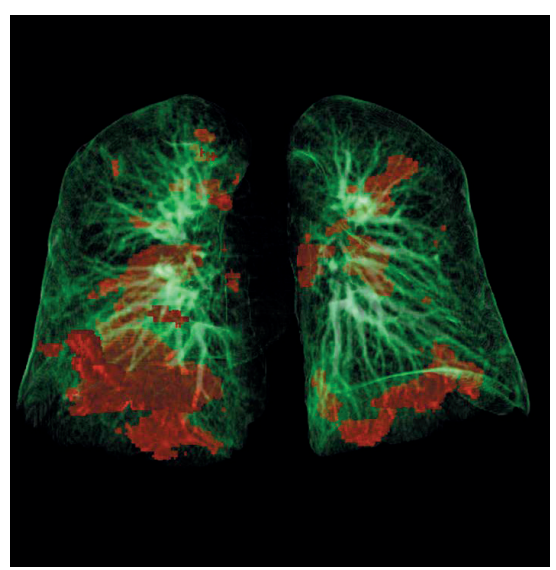

After 6 weeks

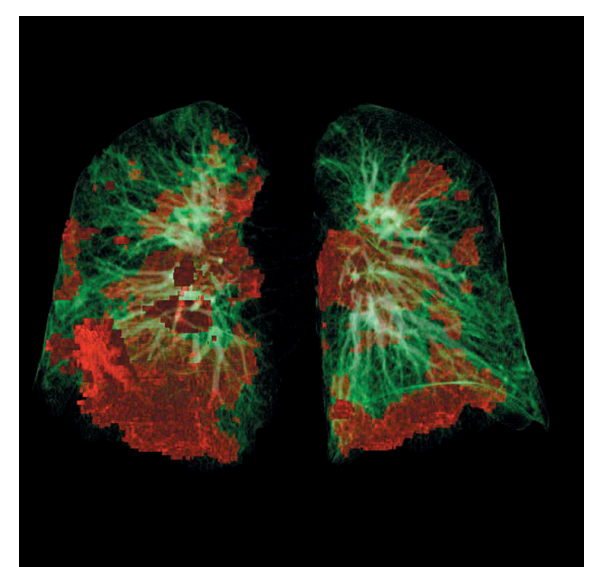

After 12 weeks

Figure 1. Pulmonary scan demonstrating changes in the lung in Prolonged COVID-19 syndrome observed in a 50-year-old patient. Lung damage (red color) decreases over time, but does not disappear completely. 
with computed tomography of the lung, precise functional examination of breathing (spirometry and plethysmography), combined with a study of diffusion capacity. A precise assessment of the condition of patients with residual symptoms after acute COVID-19 is desirable, to be carried out on the third month after the onset of symptoms.

The involvement of other organs and systems requires the assessment of a specialist and the conduct of additional imaging and functional studies such as echocardiography, imaging of the brain and laboratory studies of the cerebrospinal fluid, electroencephalography, ultrasound of the kidneys, etc.

Therapy of prolonged COVID-19 syndrome requires an experienced multidisciplinary team, individualized approach and prolonged respiratory rehabilitation. The follow-up of the sick should mainly involve general practitioners, and although pulmonologists are most involved in this process, due to the most serious affect of the respiratory system, expert advice from neurologists, psychiatrists and psychologists, cardiologists, gastroenterologists and nephrologists will also be required, which will face the most common consequences of COVID-19.

\section{POST-ACUTE HYPERINFLAMIMATORY DISEASE}

Within the course of post-acute or prolonged COVID-19, on average about 2-5 weeks after the acute phase of the disease, after cleansing the body from the virus, in children (more often) and adults (less often) develops the rare multisystem hyperinflammatory syndrome affecting organs and systems left unaffected during the acute phase. It is believed that at the base of the syndrome lies dysregulation of the immune response. In patients, symptoms develop from the cardiovascular system, gastrointestinal tract, skin and mucous membranes, manifestations that are close to Kawazaki's disease. Elevated inflammatory markers (C-reactive protein, ferritin), coagulopathy (D-dimmer), increased levels of cardiac markers (troponin) are recorded. These patients were almost without exception positive for SARS-CoV-2 antibodies and most with a negative RT-PCR test. Manifestations in adults are more colorful than in children due to the many accompanying diseases that contribute to clinical manifestations $(22,23)$.

\section{OTHER MANIFESTATIONS OF POST-ACUTE AND PROLONGED COVID-19}

In the period after acute COVID-19, symptoms from other organs and systems are also observed (9): (i) gastrointestinal - abdominal pain, appetite and disgust with food, nausea, and diarrhea, (ii) musculoskeletal - muscle and joint pain, (iii) ears, nose and throat - tinnitus and pain, throat irritation, dizziness, loss of taste and sense of smell, and (iv) skin rashes.

\section{REHABILITATION OF PATIENTS WITH PROLONGED COVID-19}

In patients after severely leaked COVID-19, after treatment in an intensive care unit and those with pronounced symptoms after the acute phase of the disease, hospital and post-hospital rehabilitation is necessary. It should start early while patients are still in the hospital (early rehabilitation), continuing in the form of outpatient rehabilitation in pulmonary rehabilitation units.

After a spent coronavirus infection, the suitability of patients to conduct rehabilitation events requires that the state of the respiratory system and the patient's blood circulation is stable without the danger of aggravation of the available complaints.

In patients with COVID-19 and significant residual symptomatology with a characteristic of prolonged COVID-19, who have had a complicated course with pronounced respiratory failure, complicated breathing, a longer period of respiratory recusal, chronic lung diseases or affecting the respiratory musculature, early respiratory rehabilitation and evaluation of each patient for his oxygen needs at rest and after loading is necessary.

In patients with COVID-19 and with prolonged COVID-19 with severe damage to the central and/or peripheral nervous system, early neurological and neurosurgical rehabilitation should be carried out. In the presence of secondary emotional disturbances, psychiatric/psychotherapeutic treatment should be initiated (24).

\section{DISCLOSURE STATEMENT}

The author declares that he has no conflicts of interest concerning the present Dance Round article.

\section{ACKNOWLEDGMENTS}

The author appreciates the work of all colleagues at his clinic involved in the ongoing study on prolonged and postCOVID-19 syndrome.

\section{REFERENCES}

1. Goërtz YMJ, Van Herck M, Delbressine JM, Vaes AW, Meys R, Machado FVC, et al. Persistent symptoms 3 months after a SARS-CoV-2 infection: the postCOVID-19 syndrome? ERJ Open Res 2020; 00542-02020. [DOI: $10.1183 / 23120541.00542-2020]$ 
2. Li L-Q, Huang T, Wang Y-q, Wang Z-p, Liang Y, Huang T-b, et al. COVID-19 patients' clinical characteristics, discharge rate, and fatality rate of meta-analysis. $J \mathrm{Med}$ Virol 2020; 92(6): 577-583. [DOI: 10.1002/jmv.25757]

3. Guan WJ, Ni ZY, Hu Y, Liang WH, Ou CQ, He JX, et al. Clinical Characteristics of Coronavirus Disease 2019 in China. N Engl J Med 2020; 382(18): 1708-1720. [DOI: 10.1056/NEJMoa2002032]

4. Docherty AB, Harrison EM, Green CA, Hardwick HE, Pius R, Norman L, et al. Features of 20133 UK patients in hospital with covid-19 using the ISARIC WHO Clinical Characterisation Protocol: prospective observational cohort study. Br Med J 2020; 369: m1985. [DOI: 10.1136/ bmj.m1985]

5. Lechien JR, Chiesa-Estomba CM, Place S, Van Laethem Y, Cabaraux P, Mat Q, et al. Clinical and epidemiological characteristics of 1420 European patients with mild-to-moderate coronavirus disease 2019. J Intern Med 2020; 288(3): 335-344. [DOI: 10.1111/joim.13089]

6. Carfî A, Bernabei R, Landi F, Group ftGAC-P-ACS. Persistent Symptoms in Patients After Acute COVID-19. $J$ Am Med Assoc 2020; 324(6): 603-605. [DOI: 10.1001/ jama.2020.12603]

7. Greenhalgh T, Knight M, A'Court C, Buxton M, Husain L. Management of post-acute covid-19 in primary care. Br Med J 2020; 370: m3026. [DOI: 10.1136/bmj. $\mathrm{m} 3026$ ]

8. Carvalho-Schneider C, Laurent E, Lemaignen A, Beaufils E, Bourbao-Tournois C, Laribi S, et al. Follow-up of adults with noncritical COVID-19 two months after symptom onset. Clin Microbiol Infect 2020. [DOI: 10.1016/j. cmi.2020.09.052]

9. NICE. COVID-19 rapid guideline: managing the longterm effects of COVID-19. In: NICE guideline. London, UK; 2020. p. 1-35.

10. Tenforde MW, Kim SS, Lindsell CJ, Billig Rose E, Shapiro NI, Files DC, et al. Symptom Duration and Risk Factors for Delayed Return to Usual Health Among Outpatients with COVID-19 in a Multistate Health Care Systems Network - United States, March-June 2020. MMWR Morb Mortal Wkly Rep 2020; 69(30): 993-998. [DOI: 10.15585/ mmwr.mm6930e1]

11. Halpin SJ, McIvor C, Whyatt G, Adams A, Harvey $\mathrm{O}$, McLean L, et al. Postdischarge symptoms and rehabilitation needs in survivors of COVID-19 infection:
A cross-sectional evaluation. $J$ Med Virol 2020: 1-10. [DOI: 10.1002/jmv.26368]

12. Rajpal S, Tong MS, Borchers J, Zareba KM, Obarski TP, Simonetti OP, et al. Cardiovascular Magnetic Resonance Findings in Competitive Athletes Recovering From COVID-19 Infection. J Am Med Assoc Cardiology 2020: 1-3. [DOI: 10.1001/jamacardio.2020.4916]

13. Zubair AS, McAlpine LS, Gardin T, Farhadian S, Kuruvilla DE, Spudich S. Neuropathogenesis and Neurologic Manifestations of the Coronaviruses in the Age of Coronavirus Disease 2019: A Review. J Am Med Assoc Neurology 2020; 77(8): 1018-1027. [DOI: 10.1001/ jamaneurol.2020.2065]

14. Galea S, Merchant RM, Lurie N. The Mental Health Consequences of COVID-19 and Physical Distancing: The Need for Prevention and Early Intervention. JAMA Intern Med 2020; 180(6): 817-818. [DOI: 10.1001/ jamainternmed.2020.1562]

15. Hampshire A, Trender W, Chamberlain SR, Jolly A, Grant JE, Patrick F, et al. Cognitive deficits in people who have recovered from COVID-19 relative to controls: An $\mathrm{N}=84,285$ online study. medRxiv 2020. [DOI: 10.1101/2020.10.20.20215863]

16. Cai X, Hu X, Ekumi IO, Wang J, An Y, Li Z, et al. Psychological Distress and Its Correlates Among COVID-19 Survivors During Early Convalescence Across Age Groups. Am J Geriatr Psychiatr 2020; 28(10): 10301039. [DOI: 10.1016/j.jagp.2020.07.003]

17. Zhao YM, Shang YM, Song WB, Li QQ, Xie H, Xu QF, et al. Follow-up study of the pulmonary function and related physiological characteristics of COVID-19 survivors three months after recovery. Clin Med 2020; 25: 100463. [DOI: 10.1016/j.eclinm.2020.100463]

18. Huang Y, Tan C, Wu J, Chen M, Wang Z, Luo L, et al. Impact of coronavirus disease 2019 on pulmonary function in early convalescence phase. Respir Res 2020; 21(1): 163. [DOI: 10.1186/s12931-020-01429-6]

19. Wong AW, Shah AS, Johnston JC, Carlsten C, Ryerson CJ. Patient-reported outcome measures after COVID-19: a prospective cohort study. Eur Respir J 2020: 2003276. [DOI: 10.1183/13993003.03276-2020]

20. NIH. Coronavirus Disease 2019 (COVID-19) Treatment Guidelines. In: NIH Guidelines, 2020. p. 1-251.

21. Berlit P. S1-Leitlinie: Neurologische Manifestationen bei COVID-19. DGNeurologie 2020; 3(6): 495-519. [DOI: 10.1007/s42451-020-00254-x] 
22. Abrams JY, Godfred-Cato SE, Oster ME, Chow EJ, Koumans EH, Bryant B, et al. Multisystem Inflammatory Syndrome in Children Associated with Severe Acute Respiratory Syndrome Coronavirus 2: A Systematic Review. J Pediatr 2020; 226: 45-54 [DOI: 10.1016/j. jpeds.2020.08.003]

23. Morris SB, Schwartz NG, Patel P, Abbo L, Beauchamps $\mathrm{L}$, Balan $\mathrm{S}$, et al. Case Series of Multisystem
Inflammatory Syndrome in Adults Associated with SARS-CoV-2 Infection - United Kingdom and United States, March-August 2020. MMWR Morb Mortal Wkly Rep 2020; 69(40): 1450-1456. [DOI: 10.15585/mmwr. mm6940e1]

24. Kluge S. et al.S2k-Leitlinie - Empfehlungen zur stationären Therapie von Patienten mit COVID-19. AWMF 2020; Register-Nr. 113/001: 1-52. 